\title{
Immunochemical studies of Aspergillus fumigatus mycelial antigens by polyacrylamide gel electrophoresis and Western blotting techniques
}

\author{
Veronica M. Hearn, ${ }^{1 *}$ Elaine V. Wilson, ${ }^{1}$ J.-P. Latgé ${ }^{2}$ and D. W. R. Mackenzie \\ ${ }^{1}$ Mycology Reference Laboratory, Central Public Health Laboratory, 61 Colindale Avenue, London NW9 5HT, UK \\ ${ }^{2}$ Unité de Mycologie, Institut Pasteur, 25-28 rue du Dr Roux, 75724 Paris Cedex, France
}

(Received 6 February 1990; revised 12 April 1990; accepted 23 April 1990)

\begin{abstract}
Differences were detectable among strains of the opportunist fungal pathogen Aspergillus fumigatus when watersoluble (WS) preparations were analysed by combined SDS-PAGE and Western blotting procedures. A wide range of molecules of apparent molecular masses from approximately 20 to $>100 \mathrm{kDa}$ showed specific binding to antibodies raised in rabbits to $A$. fumigatus wall and cytoplasmic components. The ability to bind antibody was markedly reduced by treatment of these antigens with sodium periodate or with specific proteases or glucanases. Pretreatment of blotted antigens with either concanavalin A (ConA) or wheat germ agglutinin (WGA) did not, however, inhibit subsequent antibody binding. The antigens of subfractions prepared from a single strain of $A$. fumigatus WS material were also susceptible to periodate oxidation and enzymic hydrolysis. Slight crossreactivity was apparent when crude preparations of cellular or culture filtrate antigens, used in this laboratory to detect antibodies to Candida albicans, Coccidioides immitis and Cryptococcus neoformans, were probed with hyperimmune rabbit antisera to $A$. fumigatus. Efforts were made to characterize the WS preparations of $A$. fumigatus, used as diagnostic antigens in many laboratories. The electrophoretically separated antigenic moieties were shown to be predominantly glycoproteins. Binding of cytoplasmic antigens to antibodies raised to wall material showed the presence of many common components in both wall and cytosol. Antiserum to wall components revealed most differentiation among $\boldsymbol{A}$. fumigatus strains.
\end{abstract}

\section{Introduction}

Glycoprotein molecules obtained from mycelium of the opportunist fungal pathogen Aspergillus fumigatus have been shown to possess high immunological reactivity when tested in immuno-electrophoresis and ELISA systems (Wilson \& Hearn, 1983; Schønheyder \& Andersen, 1984; Harvey \& Longbottom, 1986; Wilson et al., 1987). Some of these molecules have been partially characterized by size, by their ability or inability to bind to ConA, by possession of a specific function, e.g. enzymic activity and by their staining reactions on SDSPAGE (Calvanico et al., 1981; Schønheyder \& Andersen, 1984; Harvey \& Longbottom, 1986, 1987). In a recent study the chemical composition of partially purified fractions of $A$. fumigatus prepared in this laboratory has been analysed (Hearn et al., 1989). In the present study, these electrophoretically separated and blotted molecules of $\boldsymbol{A}$. fumigatus WS preparations and

\footnotetext{
Abbreviations: ConA, concanavalin A; WGA, wheat germ agglutinin; WS, water soluble (fraction).
}

subfractions have been probed with anti-Aspergillus sera raised in rabbits to wall and cytoplasmic material. The effects of sugar oxidation, lectin binding and enzymic hydrolysis on the ability of these antigens to bind specific antibody has been monitored in an attempt to identify the immunoreactive components of these molecules.

\section{Methods}

Water-soluble antigens from $A$. fumigatus mycelium. WS extracts were prepared from the mycelium of $A$. fumigatus strain nos NCPF 2140 , CBS 192.65, DAL. IP, DUV.IP, 152. WS and B 614B grown in neutral glucose peptone broth at $25^{\circ} \mathrm{C}$; the time of incubation varied (depending on the rate of growth of the different isolates) from 3-4 d (Debeaupuis et al., 1990). The mycelium was harvested by filtration, washed and stored at $-20^{\circ} \mathrm{C}$, at least overnight. The mycelial pad was suspended in $50 \mathrm{mM}-\mathrm{NH}_{4} \mathrm{HCO}_{3}$ at $\mathrm{pH} 8.0$ and physically ruptured with Ballotini beads at $5^{\circ} \mathrm{C}$ in a 'Dynomill' cell disintegrator (Glen Creston, $\mathrm{UK}$ ). Soluble constituents were separated from the insoluble residue by filtration and centrifugation and concentrated against polyethylene glycol 6000 to give the WS fraction (Wilson \& Hearn, 1983). An additional $A$. fumigatus strain (NCPF 2109) was used in the preparation of subfractions of WS material. Growth conditions were similar to those used above except the incubation temperature was $30^{\circ} \mathrm{C}$. These 
conditions, after a $3 \mathrm{~d}$ period, gave an adequate yield of mycelium (Proctor, 1976).

Two fractions were separated from the WS material (NCPF 2109) on the basis of their attachment or non-attachment to ConA-Sepharose (Pharmacia). Protein-rich material $(\mathrm{P} / \mathrm{C}=2 \cdot 5)$, which did not bind to ConA, was designated the UBF fraction. Carbohydrate-rich material $(\mathrm{P} / \mathrm{C}=0 \cdot 2)$, which eluted from the column with methyl $\alpha-\mathrm{D}-\mathrm{manno}-$ pyranoside, was designated the BF fraction (Wilson \& Hearn, 1983).

Two additional fractions were separated from the WS material (NCPF 2109) by salt fractionation. Material which was insoluble in the range $50-75 \%$ (saturation) ammonium sulphate (AS75) and that which was non-precipitable in a saturated solution of the salt (ASS) were retained and treated as already described (Hearn et al., 1980).

Candida albicans antigen. The antigen was obtained from a $3 \mathrm{~d}$ growth of the organism (strain no. NCPF 3153) cultured in neutral glucose peptone broth. The yeast cells were disrupted and, following centrifugation, the supernatant was used as crude diagnostic antigen.

Cryptococcus neoformans antigen. The organism (strain no. NCPF 3166) was grown (2 d) at $30^{\circ} \mathrm{C}$ in neutral glucose peptone medium. The cells were treated overnight with $0.5 \%$ formaldehyde in phosphate-buffered saline, pH 7.3 (PBS; Oxoid) and washed and stored at $4{ }^{\circ} \mathrm{C}$ as a $20 \%$ suspension (packed cell volume) in PBS.

Coccidioides immitis antigen. Mycelial phase culture filtrate antigen was purchased from Immuno-Mycologics (USA).

Production of rabbit antisera. Antiserum was obtained by hyperimmunization of New Zealand White rabbits with the WS fraction of NCPF 2109 strain of $A$. fumigatus. This fraction (at $60 \mathrm{mg} \mathrm{ml}^{-1}$ ) was mixed with an equal volume of Freund's incomplete adjuvant and $1 \mathrm{ml}$ was inoculated subcutaneously at weekly intervals for 6-8 weeks.

For predominantly carbohydrate antigens (cell wall and the BF fraction) the following protocol was used. Antigen (10 mg dry wt) was mixed with an equal volume of Freund's complete adjuvant and injected intramuscularly into a buttock; $10 \mathrm{~d}$ later the same amount of antigen was mixed with an equal volume of Freund's incomplete adjuvant and injected into the other buttock; $10 \mathrm{~d}$ later the same level of antigen with Freund's incomplete adjuvant was injected into several subcutaneous sites; $10 \mathrm{~d}$ later $5 \mathrm{mg}$ antigen was given intravenously without adjuvant; 1 week later the animal was test-bled. Sometimes another intravenous injection was required to obtain a serum of adequate titre.

The protocol used for protein-enriched antigens (cytoplasm and the UBF fraction) was as follows. Antigen ( $0.5 \mathrm{mg}$ dry wt) was mixed with an equal volume of Freund's complete adjuvant and injected intramuscularly into a buttock; $10 \mathrm{~d}$ later a similar injection was made into the other buttock. The rabbits were left for 5 months and then inoculated subcutaneously in many sites with $0.5 \mathrm{mg}$ antigen mixed with Freund's incomplete adjuvant; after $10 \mathrm{~d}$ the animals were given $0.1 \mathrm{mg}$ antigen intravenously without adjuvant; a test-bleed was taken $10 \mathrm{~d}$ later. Sometimes an additional intravenous injection was required (Hearn \& Mackenzie, 1980a; John et al., 1984).

' $\mathrm{C}$ ' (chymotryptic)-antigen has been described by Tran van $\mathrm{Ky}$ et al. (1966) as specific for serodiagnosis of aspergillosis caused by $A$. fumigatus. C-lines revealed by immuno-electrophoresis using polyvalent $A$. fumigatus antigen and a monospecific rabbit antiserum in six separate gels were carefully cut out, pooled, and homogenized in $2 \mathrm{ml}$ of saline $(0.85 \% \mathrm{NaCl})$. An equal volume of Freund's complete adjuvant was added and the suspension was injected at about 60 different subcutaneous sites in a New Zealand White rabbit. An additional single injection of $1 \mathrm{ml}$ of Bordetella pertusis vaccine (Wellcome) was administered intramuscularly to boost antibody production. The animal was test-bled at intervals and exsanguinated after 9 months. Specificity of the reactions was checked by immuno-electrophoresis against the same polyvalent antigen (de Magaldi \& Mackenzie, 1984). The antiserum to $A$. fumigatus ' $\mathrm{C}$ '-antigen was a generous gift from Professor S. de Magaldi (Universidad Central de Venezuela, Caracas). WS/TE antigen was obtained by extracting crude wall material with Triton X-100. Subsequent removal of the detergent yielded an insoluble residue and a WS fraction, i.e. WS/TE, with antigenic activity (Hearn \& Mackenzie, $1980 \mathrm{~b}$ ). Antiserum to this antigen was prepared in a manner analogous to that used for ' $C$ '-antigen. It was electrophoresed against rabbit antiserum to crude walls, and the only two dominant precipitin arcs were cut out of the gel for injection into rabbits. Serum was collected from rabbits before injection of antigens for use as a negative control.

Preparation of an IgG fraction. Rabbit antisera were treated with caprylic acid (Steinbuch \& Audran, 1969). The IgG component (and a portion of the $\operatorname{IgA}$ ) remained in the supernatant; the precipitate was discarded.

Chemical analyses. Total protein of $A$. fumigatus fractions was estimated by the dye-binding method of Read \& Northcote (1981) with BSA as a standard. Total neutral sugar was determined by the method of Dubois et al. (1956) with glucose as a standard.

SDS-PAGE. Vertical PAGE using gel slabs $1.5 \mathrm{~mm}$ thick with a separating gel of either $10 \%$ or a gradient of $5-15 \%(w / v)$ polyacrylamide containing $0 \cdot 1 \%$ SDS was done according to Laemmli (1970). Samples for analysis were taken up in 'dissolving buffer' $(2 \%, w / v$, SDS; $10 \%, \mathrm{v} / \mathrm{v}$, glycerol; $5 \%, \mathrm{v} / \mathrm{v}, \beta$-mercaptoethanol; plus a trace of bromophenol blue in $64 \mathrm{~mm}$-Tris/ $\mathrm{HCl}$ buffer, $\mathrm{pH} \mathrm{6.8)} \mathrm{and} \mathrm{boiled} \mathrm{for}$ $3 \mathrm{~min}$ before application of a $50 \mu \mathrm{l}$ aliquot to each well. When WS preparations from different isolates of $\boldsymbol{A}$. fumigatus were compared, loading was uniform at $35 \mu \mathrm{g}$ protein per track. Loading of $A$. fumigatus fractions was done on the basis of the optimum number of bands visualized on stained gels and blots stained with Indian ink. This procedure was followed because the fractions varied considerably in the proportion of protein and carbohydrate present in each. Whole-cell Cryptococcus antigen was boiled for $10 \mathrm{~min}$ as a $10 \%(\mathrm{w} / \mathrm{v})$ suspension in dissolving buffer and, after centrifugation, the supernatant was used at a volume which gave the maximum number of bands. For $C$. albicans WS antigen the maximum number of identifiable bands was seen at a loading of $5 \mu \mathrm{g}$ protein per well; for $C$. immitis the loading was $18 \mu \mathrm{g}$ protein per well. In tests with heterologous antisera, the gel loading of each of the above antigen preparations was increased 3-fold. Gels were subjected to electrophoresis at a current of approximately $30 \mathrm{~mA}$ until the dye marker had reached the bottom of the gel. High molecular mass protein standards $(14.3-200 \mathrm{kDa}, \mathrm{Gibco})$ were electrophoresed in parallel (Hearn et al., 1989). Methyl green solution $(2 \mu 1 ; 0 \cdot 1 \%$ in water containing $10 \%$ glycerol) was introduced into each well of the stacking gel $10 \mathrm{~min}$ before the end of the run. The dye transblotted to nitrocellulose and served to locate sample tracks (Ramirez et al., 1983).

Protein and glycoprotein detection. Separated components were stained for protein using the Bio-Rad silver stain and counterstained in $0.1 \%$ Coomassie Brilliant Blue R-250 in $25 \%(\mathrm{v} / \mathrm{v})$ methanol and $7.5 \%$ $(\mathrm{v} / \mathrm{v})$ acetic acid for $1 \mathrm{~h}$ at room temperature and destained in the same solvent without dye (Dzandu et al., 1984).

Electroblotting. The electrophoretically separated proteins and glycoproteins together with protein standards were electro-transferred to nitrocellulose membranes (Hybond C, Amersham) in a transblotting chamber (LKB2005 Transphor) using the method of Towbin et al. (1979). Transfer was done in $25 \mathrm{~mm}$-Tris/192 mM-glycine (pH 8.3) containing $20 \%$ methanol at $80 \mathrm{~V}, 350 \mathrm{~mA}$ for $2 \mathrm{~h}$ at $8{ }^{\circ} \mathrm{C}$. Blots were stained with Indian ink (0.1\% in PBS-Tween 20) as described by Hancock \& Tsang (1983), except the time of incubation was reduced to $1 \mathrm{~h}$. 
Reactivity of antigens. Sera were incubated with transblotted antigens following saturation of the free binding sites on the membrane with PBS containing $0.15 \%$ Tween 20 (PBS-T, blocking buffer) for $1 \mathrm{~h}$ at room temperature. Partially purified IgG fractions, prepared from rabbit, were used at a dilution of $1: 800$ in PBS-T; incubation was either for $1.5 \mathrm{~h}$ at room temperature or overnight at $4{ }^{\circ} \mathrm{C}$ with continuous agitation in each case. Blots were subsequently exposed to swine antirabbit IgG conjugated to peroxidase. The peroxidase-IgG conjugate was obtained from Dako (Denmark) and was used at a 1:1000 dilution in blocking buffer for $1 \mathrm{~h}$ at room temperature. Antigen/antibody complexes were localized by staining for peroxidase activity with $3,3^{\prime}$ diaminobenzidine as substrate. The reaction was stopped with tap water after a maximum incubation time of $10 \mathrm{~min}$.

Inhibition of antigen/antibody binding by lectins. Blotted fractions were exposed to either ConA or WGA, each at $100 \mu \mathrm{g} \mathrm{ml}^{-1}$, for $2 \mathrm{~h}$ at room temperature (Monk, 1987). The blots were washed and subsequently incubated with an antiserum, raised in rabbits to the WS fraction, used at a dilution of 1:800 in the appropriate blocker. Because ConA has the ability to bind free peroxidase molecules, a goat anti-rabbit IgG fraction conjugated to alkaline phosphatase (Sigma) was selected for use in these experiments. Bound IgG was detected using freshly prepared filtered mixtures of equal volumes of naphthol ASMX phosphate (Sigma; $0.4 \mathrm{mg} \mathrm{ml}^{-1}$ in distilled water) and fast red TR salt (Sigma; $6 \mathrm{mg} \mathrm{ml}^{-1}$ in $200 \mathrm{~mm}-\mathrm{Tris} / \mathrm{HCl}, \mathrm{pH} 8.2$ ) for a maximum of $15 \mathrm{~min}$ at room temperature.

Periodate oxidation of extracts. A. fumigatus WS preparations and fractions were treated with $50 \mathrm{mM}$-sodium periodate in acetate buffer (10 mM, pH 4.5). Incubation was in the dark at room temperature for either 2 or $18 \mathrm{~h}$. Subsequently, 'dissolving buffer' was added to the samples which were boiled for $3 \mathrm{~min}$ and subjected to electrophoresis. Transblotted components were incubated with antiserum prepared in rabbits immunized with WS material. Control samples without periodate were included for comparative purposes.

Incubation of extracts with hydrolytic enzymes. A. fumigatus WS preparations and fractions were incubated with different hydrolytic enzymes either prior to, or following electrophoresis and transblotting. $\alpha$-Amylase (EC 3.2.1.1) from Bacillus, lytic enzyme $L_{1}$ from Cytophaga [which contains several endo- $\beta$-1,3- and endo- $\beta$-1,4-glucanases] and Pronase, a non-specific protease from Streptomyces griseus were purchased from BDH. $\beta$-Amylase, type III from barley (EC 3.2.1.2), papain from papaya latex (EC 3.4.22.2), trypsin type I from bovine pancreas (EC 3.4.21.4) and $\alpha$-chymotrypsin type II from bovine pancreas (EC 3.4.21 .1) were obtained from Sigma. Enzymes were used at a final concentration of $0.4 \%(\mathrm{w} / \mathrm{v})$ and were used at the $\mathrm{pH}$ specified by the supplier in an appropriate buffer solution $(50 \mathrm{mM})$. Hydrolysis was allowed to proceed for $2 \mathrm{~h}$ at $37^{\circ} \mathrm{C}$. Treatment with 'dissolving buffer' and SDS-PAGE were the standard protocols described above. Where enzyme treatment preceded electrophoretic separation, duplicate gels were processed. One was stained for protein with Coomassie Blue and one was transblotted to nitrocellulose. Following transfer, any free sites on the membranes were saturated with PBS-T and the blots were then incubated with an IgG fraction, produced in rabbits immunized with WS material, at a dilution of $1: 800$. The extent to which enzyme treatment affected antigen/antibody binding was measured against controls incubated without enzyme.

Effect of enzymes on lectin binding to antigen. ConA binding. Transblotted enzyme-treated filters were placed in a solution of $3 \%$ (w/v) BSA in Tris-buffered saline (TBS; $50 \mathrm{~mm}-\mathrm{Tris} / \mathrm{HCl}, \mathrm{pH} 7.5$, $150 \mathrm{mM}-\mathrm{NaCl}$ ) for $\mathrm{l} \mathrm{h}$ at room temperature in order to saturate any free sites on the membrane. After air-drying the blot was incubated with ConA-peroxidase (Sigma) for $30 \mathrm{~min}$ at room temperature at a dilution of $2 \mu \mathrm{g} \mathrm{BSA} / \mathrm{TBS} \mathrm{ml} \mathrm{m}^{-1}$ (Hearn et al., 1989).
WGA binding. Transblotted enzyme-treated filters were placed in a blocking solution of $2 \%(\mathrm{w} / \mathrm{v})$ polyvinylpyrrolidone in TBS for $1 \mathrm{~h}$ at $40^{\circ} \mathrm{C}$. The blot was then incubated with WGA-peroxidase (Sigma) for $2 \mathrm{~h}$ at room temperature at a dilution of $2.5 \mu \mathrm{g}$ per ml of blocking buffer (Hearn et al., 1989).

\section{Results}

\section{SDS-PAGE and Western blots of A. fumigatus strains}

WS preparations from NCPF 2109 and six additional strains of $A$. fumigatus were separated by SDS-PAGE and stained for protein with Coomassie Blue (Fig. 1a). A range of molecules which differed in apparent molecular mass from $<10-97 \mathrm{kDa}$ was seen with these extracts. Two WS fractions (DUV.IP and DAL.IP) showed relatively poor staining with Coomassie Blue and were not used in subsequent experiments. The remaining preparations showed similar, but non-identical profiles. The presence of 30-40 bands in each extract make it difficult to identify individual components. However, band doublets with apparent molecular masses of 91 and $97 \mathrm{kDa}$ were present in three strains; the former component was present in all five strains. For a profile of prominent band patterns see Table 1 .

The five selected WS preparations showed a complex array of molecules when reacted with a rabbit antiserum raised to the WS-NCPF 2109 material (Fig. 1b). The majority of bands appeared common to all samples of which a few were readily identified, viz. 16, 22, 59, 71, 97 and $101 \mathrm{kDa}$. Only strain CBS 192.65 appeared to possess a reactive antigen of apparent molecular mass $=$ $110 \mathrm{kDa}$. An antigen of apparent molecular mass = $120 \mathrm{kDa}$ appeared to be unique to the B614B strain, when probed with this antiserum. The profiles given by an antiserum produced against the BF fraction looked very similar to those obtained with antiserum to WS and are not shown here.

Reactivity of these WS preparations to an antiserum raised to wall components was relatively weak. A maximum number of 20 bands could be identified in any one sample, but many of these were pale and diffuse. A number of discrete bands were readily identifiable as present in four preparations. These molecules had apparent molecular masses of 19, 25, 32 and $54 \mathrm{kDa}$. Three of five preparations had an antigen of apparent molecular mass $=91 \mathrm{kDa}$ which reacted with this antiserum. One strain (NCPF 2140) showed traces of only two bands of apparent molecular masses $=22$ and $75 \mathrm{kDa}$ (Fig. 1c).

An antiserum to the cytoplasmic constituents also reacted quite strongly with all five WS preparations (Fig. $1 d$ ). Given the multiplicity of bands, only a few dominant bands could be seen as either common or 
(a)

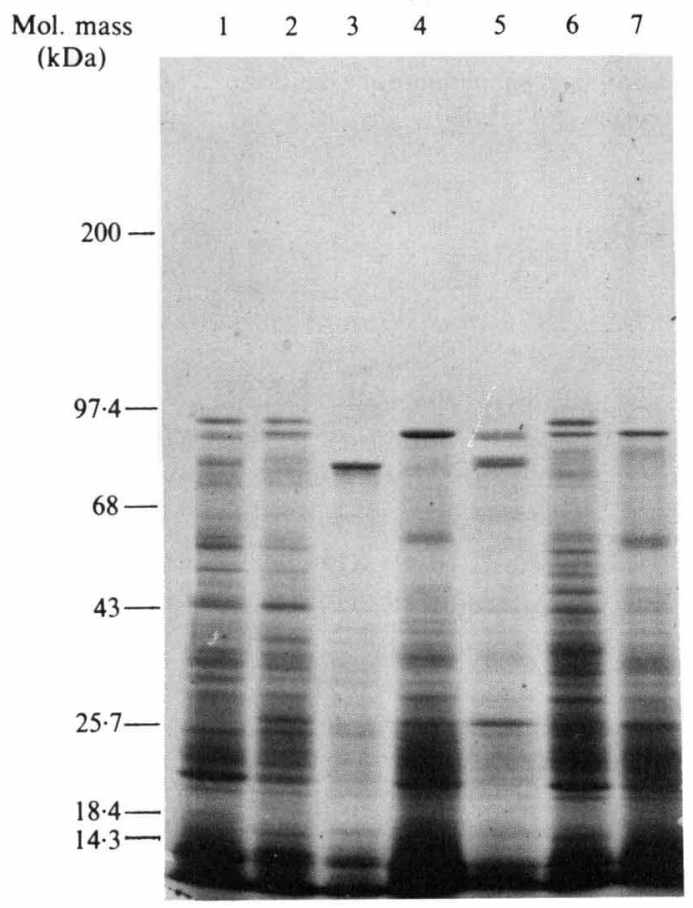

(c)

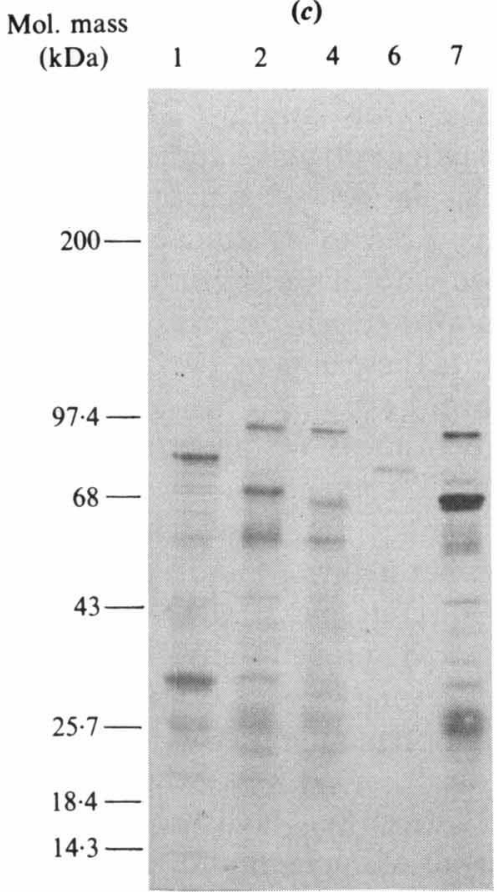
Mol. mass
(kDa)
(b)

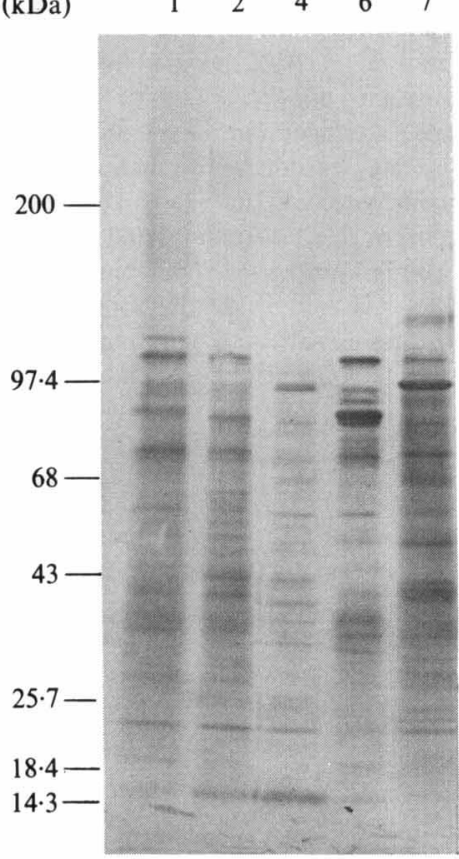
Mol. mass
$(\mathrm{kDa})$

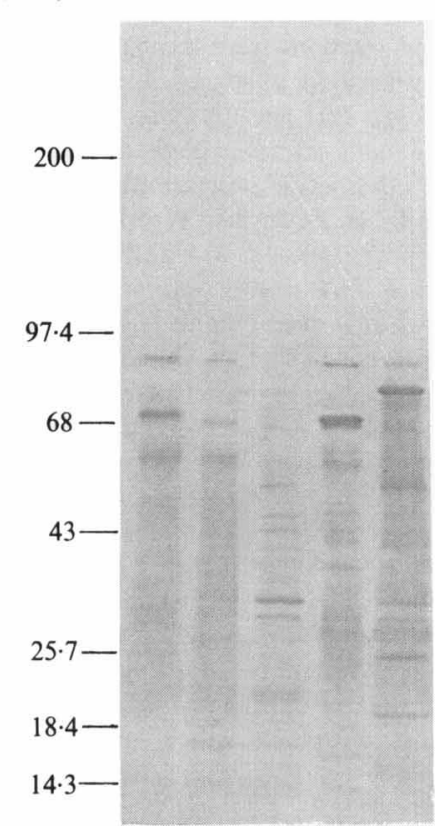

Fig. 1. SDS-PAGE on a 5-15\% gradient gel and subsequent transblotting to nitrocellulose of WS preparations from different isolates of A. fumigatus. (a) Gel stained with Coomassie Blue. (b-d) Immunoblots of WS preparations from five isolates of $A$. fumigatus exposed to antisera raised in rabbits to subcellular components of $A$. fumigatus: $(b)$ antiserum to WS material; $(c)$ antiserum to crude wall material; (d) antiserum to cytoplasmic components. Lane 1, A. fumigatus CBS 192.65; 2, NCPF 2109; 3, DUV.IP; 4, 152. WS; 5, DAL. IP; 6, NCPF 2140; 7, B614B. 
Table 1. Prominent protein and antigen bands detectable in five strains of A. fumigatus

The values refer to the total number of strains in which a band of given molecular mass was found. The strains examined were $A$. fumigatus CBS 192.65, NCPF 2109, 152. WS, NCPF 2140 and B614B.

\begin{tabular}{lcllccc}
\hline \hline & \multicolumn{2}{c}{ Stain } & \multicolumn{5}{c}{ Antiserum to: } \\
\cline { 2 - 3 } $\begin{array}{c}\text { Molecular } \\
\text { mass (kDa) }\end{array}$ & $\begin{array}{c}\text { Coomassie } \\
\text { Blue }\end{array}$ & & WS & Wall & Cytoplasm & 'C'-antigen \\
\hline 16 & 5 & 5 & 0 & $4 \dagger$ & 0 \\
$19^{*}$ & 5 & $4 \ddagger$ & $4 \dagger$ & 0 & 0 \\
22 & 5 & 5 & 1 & 5 & 0 \\
$25^{*}$ & 5 & 0 & $4 \dagger$ & 0 & 0 \\
91 & 5 & $4 \ddagger$ & 3 & $4 \ddagger$ & 0 \\
$93-95^{*}$ & 0 & 0 & 0 & 0 & $4 \ddagger$ \\
97 & 3 & 5 & 0 & 0 & 0 \\
\hline \hline
\end{tabular}

* Bands of these molecular masses were also found when antisera to WS, wall and ' $\mathrm{C}$ 'band components were reacted with the BF and AS75 subfractions.

$\uparrow$ Band absent from strain NCPF 2140.

$\ddagger$ Band absent from strain 152. WS.

unique to the different samples. An antigen of apparent molecular mass $=91 \mathrm{kDa}$ was present in all strains with the exception of 152. WS. A rabbit antiserum to 'C'-band antigen bound components with apparent molecular masses of $93-95 \mathrm{kDa}$ present in the WS preparations with the exception of one strain (152.WS) which lacked this band doublet but showed traces of bands of apparent molecular masses $=43$ and $44 \mathrm{kDa}$. These trace bands were detectable in all WS preparations (results not shown). Serum from pre-immunized rabbits showed no detectable binding to components of $A$. fumigatus WS material.

Candida albicans preparations, on electrophoretic separation, showed components with apparent molecular masses of $10-100 \mathrm{kDa}$. Approximately 20 bands were seen which stained with both silver reagent and Coomassie Blue dye. The Coccidioides immitis preparation contained 8-10 predominantly Coomassie-Bluestaining bands in the molecular mass range $18-50 \mathrm{kDa}$ with a major component of apparent molecular mass $=$ $43 \mathrm{kDa}$. The Cryptococcus neoformans detergent-solubilized extract showed mainly lower molecular mass entities which gave intense staining with silver reagent. It contained a major component of apparent molecular mass $=30 \mathrm{kDa}$. (These data are not shown.)

\section{Gel electrophoresis of A. fumigatus WS fractions}

A. fumigatus WS material together with four fractions prepared from it were subjected to SDS-PAGE: the separated components were stained for protein with a combined silver stain and Coomassie Blue reagent (Fig. $2 a$ ). The silver stain was used in an attempt to gain additional sensitivity. Each sample contained an array of molecules which ranged in apparent molecular mass from $<10$ to approximately $100 \mathrm{kDa}$. The ASS fraction contained, in addition, bands between 100-200 kDa and traces of components with apparent molecular masses in excess of $200 \mathrm{kDa}$. Most of the subunits in the ASS fraction as well as the lower molecular mass moieties of the WS ( $<34 \mathrm{kDa})$, AS75 and UBF ( $<17 \mathrm{kDa})$ fractions stained preferentially with silver. In contrast, the bulk of the components present in the BF fraction (approximately $10-81 \mathrm{kDa}$ ) bound solely to Coomassie Blue. A few of the higher molecular mass entities of the WS, AS75 and UBF fractions $(>74 \mathrm{kDa})$ also stained predominantly with Coomassie Blue (cf. Hearn et al., 1989).

\section{Electroblotting}

Proteins and glycoproteins transblotted to nitrocellulose membranes were stained with Indian ink. Results showed fractions containing 20-30 bands with patterns similar, but not identical to those given by stained gels (Fig. $2 b$ ). WS contained six readily identifiable bands or band doublets (apparent molecular masses 13, 16, 22, 36, 64 and $79-87 \mathrm{kDa}$ ). Several of these bands were present in the subfractions of WS. All fractions also showed one or more bands between $74-98 \mathrm{kDa}$. The very high molecular mass species present in the ASS fraction $(>100 \mathrm{kDa})$ failed to transblot under the experimental conditions used. 
(a)

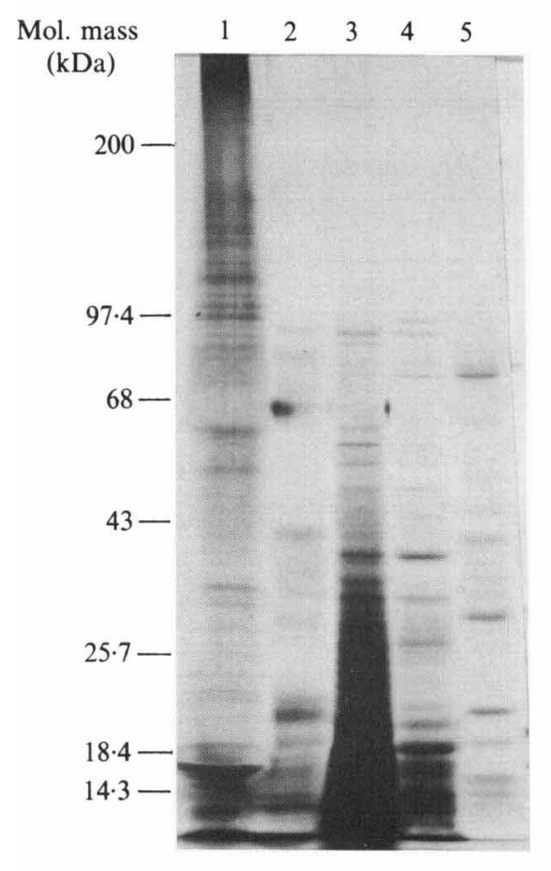

(c)

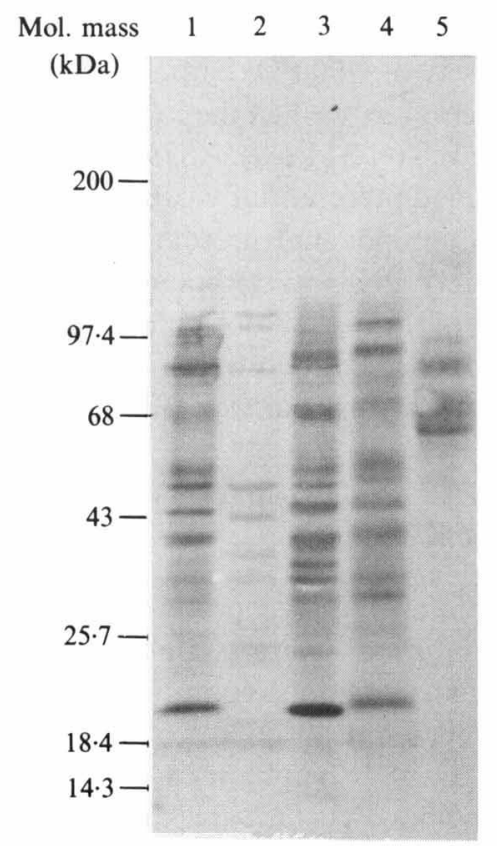

(b)

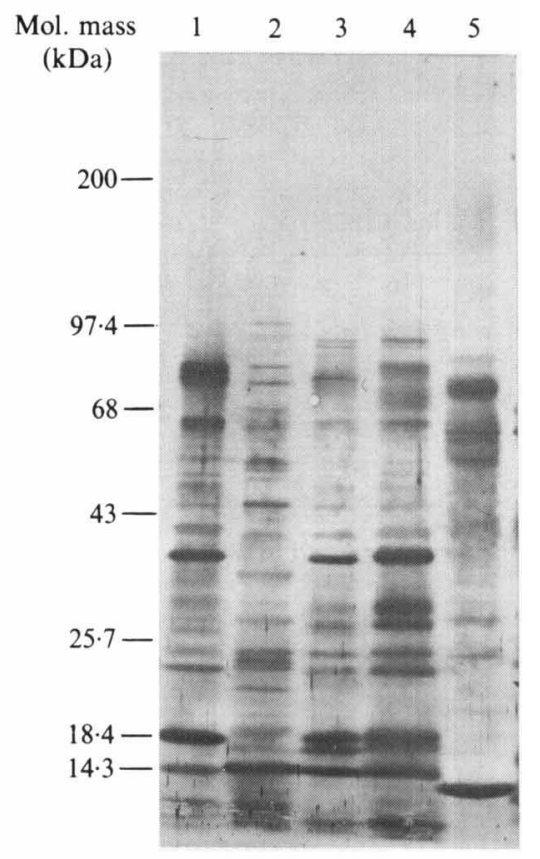

(d)

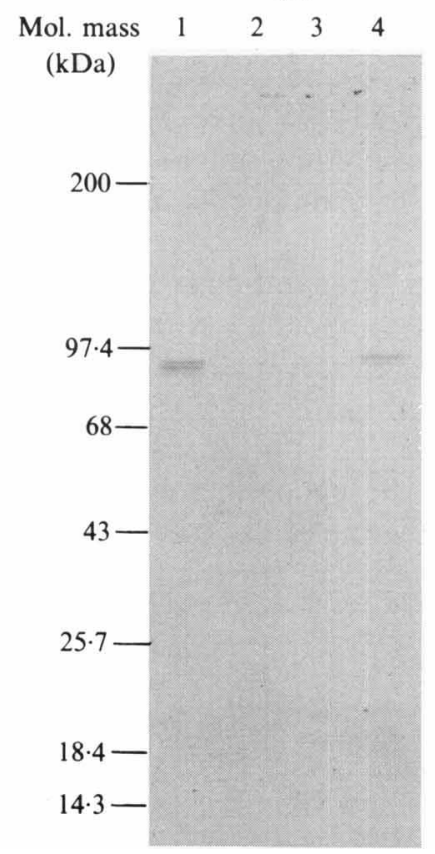

Fig. 2. SDS-PAGE on a 5-15\% gradient gel and subsequent transblotting to nitrocellulose of WS fractions from A. fumigatus mycelium. (a) Gel stained by the combined silver/Coomassie Blue procedure; (b) blot stained for protein with Indian ink. Lane 1, ASS; 2 , AS75; 3 , WS; 4, UBF; 5, BF. $(c, d)$ Blots exposed to antisera raised in rabbits to $A$. fumigatus subcellular components $[c$, WS material; $d$, 'C' (chymotryptic)-antigen]. Lane 1, WS; 2, UBF; 3, BF; 4, AS75; 5, ASS. 


\section{Blots developed with rabbit anti-A. fumigatus antibodies}

All fractions, excepting ASS, showed a complex array of molecules when reacted with a rabbit antiserum raised to the WS material (Fig. 2c). The WS fraction showed three areas of prominent reactivity, viz. bands with apparent molecular masses of 19, 37-51 and 78-100 kDa. Bands with similar molecular masses were found in fractions $\mathrm{BF}$ and AS75. Some of these bands were seen in the UBF fraction, but the reactions were much weaker. The profile given by an antiserum produced against the carbohydrate-rich BF fraction looked essentially similar except the serum lacked binding capacity towards the UBF antigenic subunits (results not shown). When compared with WS and BF antisera, those prepared to crude wall material and cytoplasmic constituents reacted more weakly and with fewer components of $A$. fumigatus. The major areas of reactivity detected were bands with apparent molecular masses of 25, 48-66 and 96-118 kDa. These two sera differed from each other mainly in their responses to the non-ConA binding, protein-rich fraction (UBF). Wall antiserum reacted minimally with UBF subunits while antiserum to cytoplasmic constituents showed a reaction similar to that given by the antiserum to WS (results not shown). A rabbit antiserum to 'C'-band (chymotryptic) antigen showed staining of one or two components of WS and AS75 with apparent molecular masses of 93-95 kDa (Fig. 2d). Other $A$. fumigatus fractions gave only trace reactivity. Sera from pre-immunized rabbits gave negative results.

\section{Cross-reactivity studies}

Antigens from Candida albicans, Coccidioides immitis and Cryptococcus neoformans displayed no binding to antibodies present in either normal rabbit serum or to antibodies raised in rabbit to $A$. fumigatus WS material. When antigen loading onto gels was increased 3-fold above that used for routine tests, $A$. fumigatus antiserum showed slight reactivity towards heterologous antigens. Two or three trace bands were found with Candida albicans and Cryptococcus neoformans and six with Coccidioides immitis transblotted antigens. These appeared not to react (except for two trace bands with Coccidioides immitis) with an antiserum raised to the $\mathrm{BF}$ fraction. Two low molecular mass entities present in all fungal classes and with apparently identical mobilities reacted with an antiserum to crude $A$. fumigatus wall material. These cross-reacting bands (apparent molecular masses $=15$ and $20 \mathrm{kDa}$ ) were even more marked when an antiserum to water-soluble $A$. fumigatus wall fraction (WS/TE) was used (results not shown).

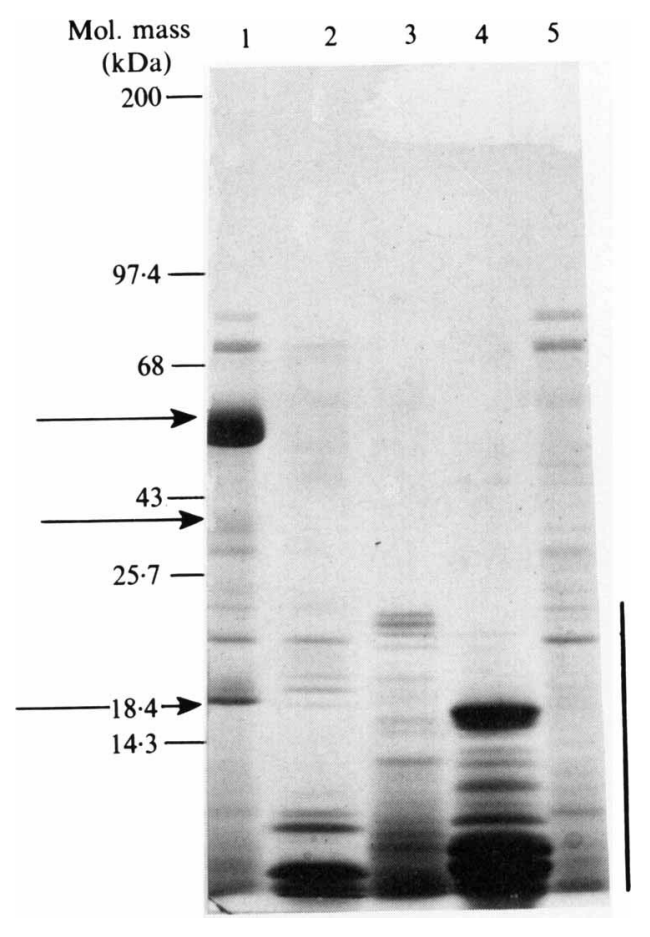

Fig. 3. SDS-PAGE on a $10 \%$ gel of $A$. fumigatus WS-NCPF 2109 preparation pretreated with selected enzymes. Lane $1, \alpha$-amylase; 2 , chymotrypsin; 3 , pronase; 4 , papain ; 5 , control WS incubated without enzyme. The gel was stained with Coomassie Blue. Arrows and the solid vertical bar indicate overlay of enzyme bands present in the incubation mixture.

\section{Effect of periodate oxidation on antigen/antibody binding}

The WS extracts obtained from four strains of $A$. fumigatus were tested for their sensitivity to sodium periodate. Overnight incubation with 50 mM-sodium periodate abolished all antigenic activity relative to controls, which were apparently unaffected by overnight incubation in acetate buffer in the absence of periodate. The bulk of the antigenic reactivity of $A$. fumigatus WSNCPF 2109 and fractions was lost following incubation for $2 \mathrm{~h}$ with periodate. A few additional bands, mainly of apparent molecular mass in the $50 \mathrm{kDa}$ range, were lost during overnight incubation. Each fraction prepared from WS retained one component which was resistant to oxidative degradation. While these results were reproducible, it should be noted that oxidation of sugar residues prior to electrophoresis may have altered the mobilities of constituent molecules.

\section{Effect of hydrolytic enzymes on antigen/antibody binding}

Enzymes were incubated with antigens prior to analysis by SDS-PAGE and immunoblotting. Quite similar gel patterns were seen with all WS preparations, one of which is reproduced (Fig. 3). In general, WS antigens 


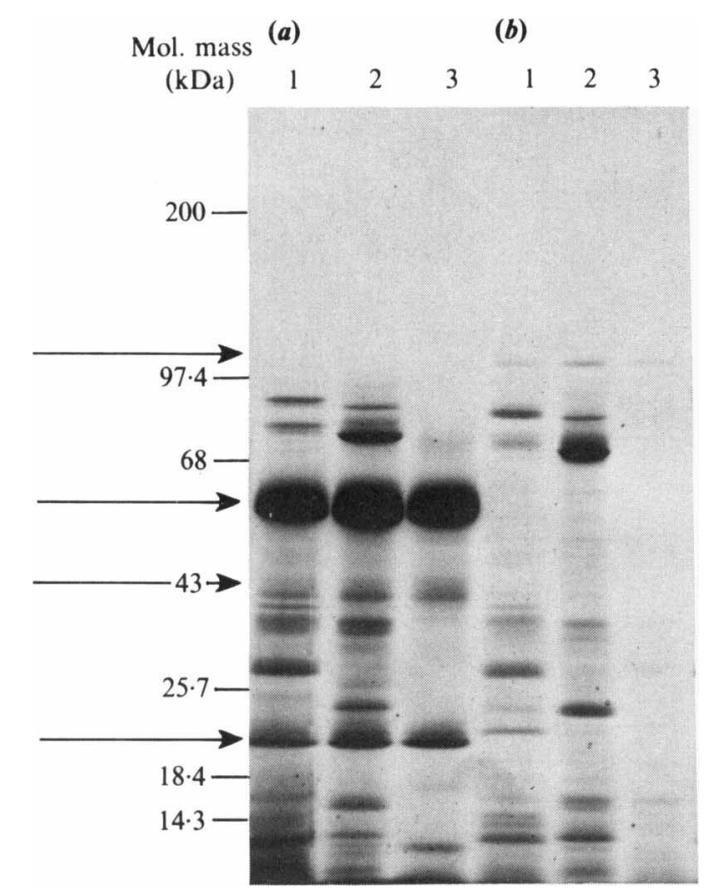

Fig. 4. SDS-PAGE on a $5-15 \%$ gradient gel of $A$. fumigatus WSNCPF 2109 and fractions pretreated with carbohydrases. (a) $\alpha-$ Amylase; (b) Cytophaga lytic enzyme. Lane 1, WS; 2, BF; 3, ASS. Arrows indicate overlay of enzyme bands present in the incubation mixture.

appeared resistant to hydrolysis by $\alpha$-amylase; the lytic enzyme from Cytophaga also had little effect (results not shown). In contrast, proteolytic enzymes, including trypsin and chymotrypsin, caused appreciable hydrolysis of all WS preparations. Chymotrypsin destroyed bands principally in the range $18-47 \mathrm{kDa}$ while traces of bands of apparent molecular masses $=47,58$ and $76 \mathrm{kDa}$ remained. Pronase and papain were the most destructive in their action, leaving only residual amounts of components of molecular mass $=58$ and $47 \mathrm{kDa}$, respectively. Protein bands from $\alpha$-amylase are indicated by arrows (Fig. 3). Bands from chymotrypsin had apparent molecular masses from $8 \mathrm{kDa}$ to the gel front while the presence of protein subunits of pronase and papain covered the range from $21 \mathrm{kDa}$ to the gel front. These effects were confirmed in blotting experiments, when enzyme-treated samples were electrophoresed, transblotted and probed with rabbit antiserum to the WS-NCPF 2109 preparation. Gel patterns were reproduced on blots, indicating that the limited hydrolysis of intact molecules of $A$. fumigatus extracts catalysed by polysaccharolytic enzymes had an equally limited effect on antigen binding sites (results not shown). Similarly, the destructive effects of proteases were mirrored by the absence of many antibody-binding antigen bands.

Carbohydrases had a limited effect on the BF fraction; dominant bands of apparent molecular masses $=71,76$

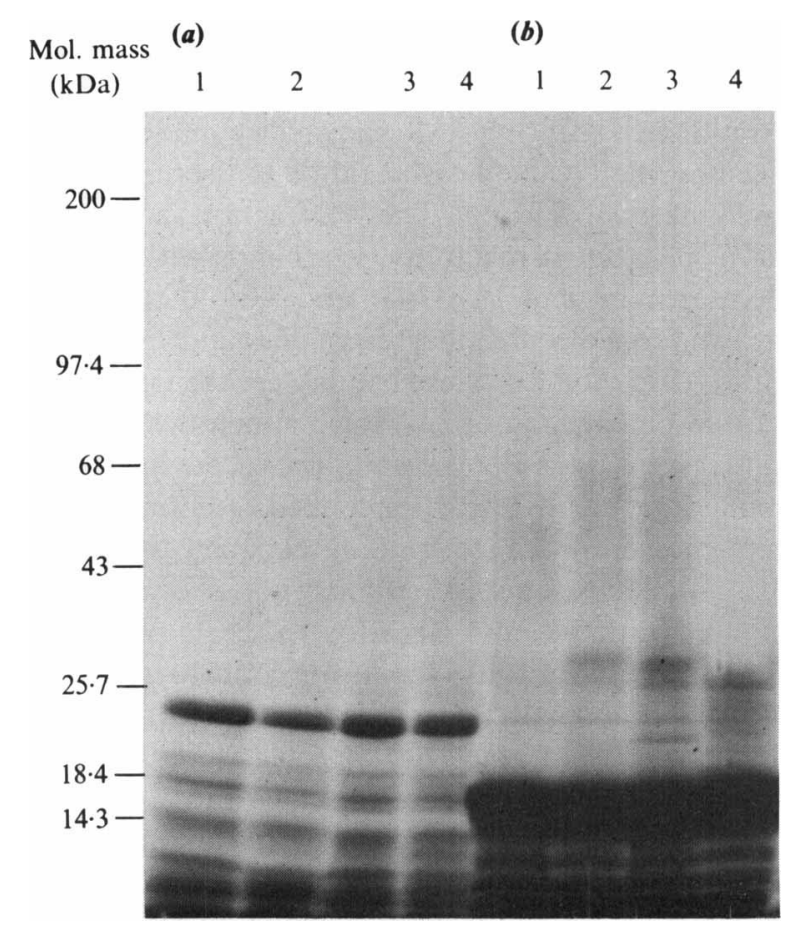

Fig. 5. SDS-PAGE on a 5-15\% gradient gel of $A$. fumigatus WSNCPF 2109 and fractions pretreated with proteases. (a) Pronase; (b) papain. Lane 1, WS; 2, BF; 3, AS75; 4, ASS. The solid vertical bar indicates overlay of enzyme bands present in the incubation mixture.

and $83 \mathrm{kDa}$ appeared entirely unaffected. The ASS fraction was more susceptible to hydrolysis, especially by the lytic enzyme from Cytophaga (Fig. 4). The antigen fractions, like WS, were most sensitive to proteolytic enzymes. The bands seen on Coomassie-Blue-stained gels were mainly due to enzyme subunits present after hydrolysis (Fig. 5).

The above procedure has the disadvantage that enzyme subunits present in the incubation mixture were visible on SDS-PAGE and obscured the full extent of antigen hydrolysis, especially in the low molecular mass range. In addition, enzyme hydrolysis may cause partial molecular destruction, resulting in altered mobilities on subsequent electrophoresis. Therefore antigens were electrophoresed, transblotted and then incubated with enzymes, prior to exposure of WS to antiserum. Of the polysaccharolytic hydrolases, the lytic enzyme from Cytophaga caused the greatest disruption to antigen/antibody binding (Fig. $6 a$ ). Antigen bands remaining after treatment had apparent molecular masses between 20 $46 \mathrm{kDa} . \alpha$-Amylase was much less disruptive while $\beta$ amylase had no discernible effect on antigen reactivity (Fig. 6b). All proteolytic enzymes caused considerable reduction in antigen/antibody binding, but the WS and antigenic fractions varied in their susceptibilities to hydrolysis. In general, the lower molecular mass species of each antigen were least affected by enzyme action. 
(a)

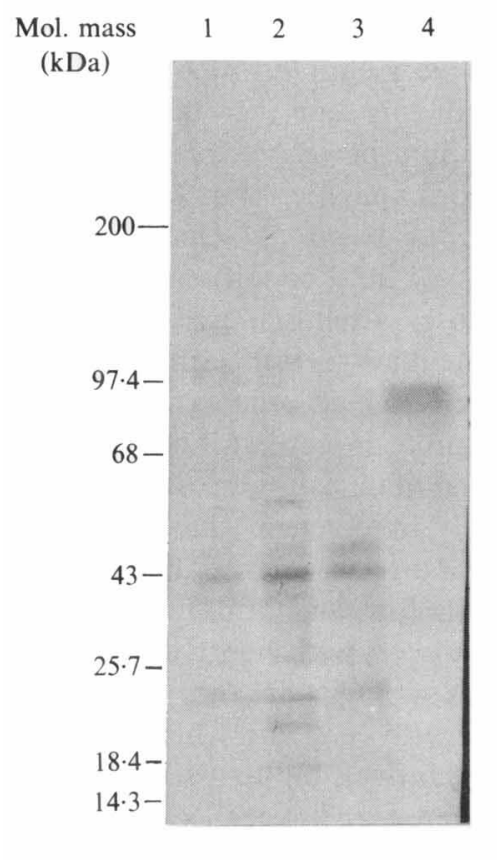

(c)

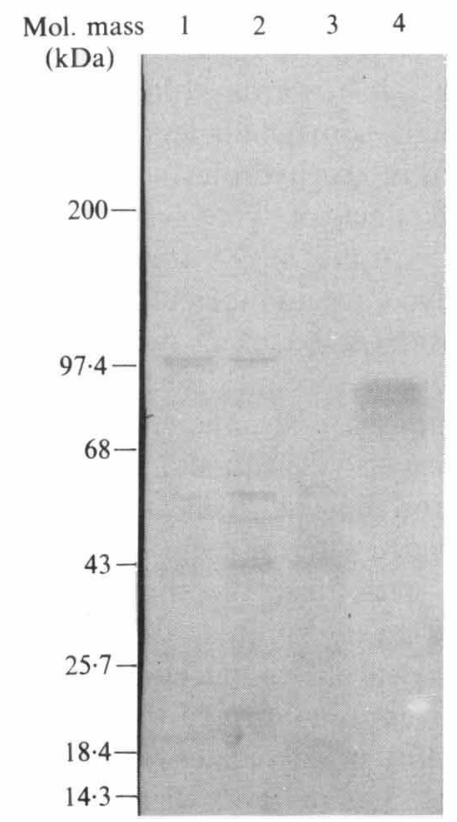

(b)

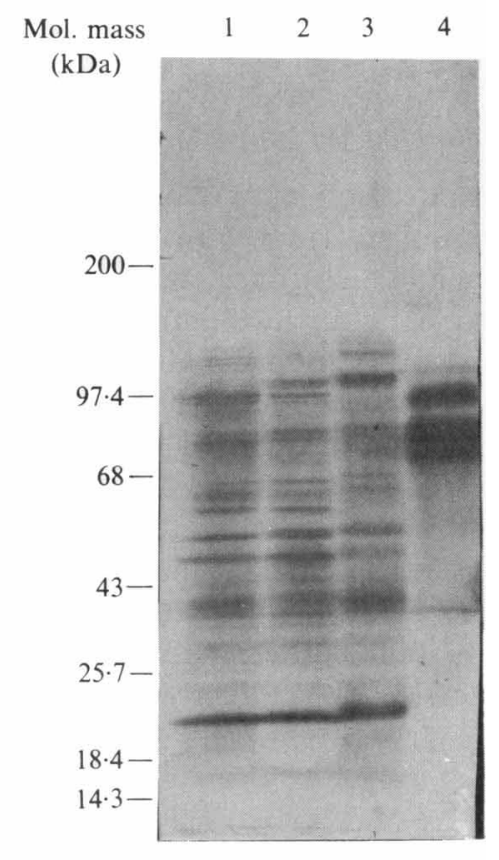

(d)

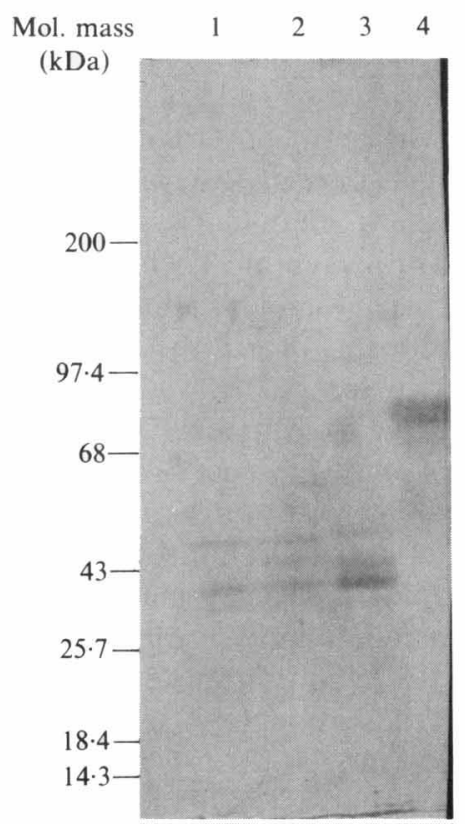

Fig. 6. Immunoblots of $A$. fumigatus antigens treated with selected enzymes before exposure to anti-Aspergillus antiserum raised in rabbits to the WS material. (a) Cytophaga L1 lytic enzyme; (b) $\beta$-amylase; (c) pronase; (d) trypsin. Lane 1 , WS; 2 , BF; 3 , AS75; 4 , ASS.

Blots obtained after pronase and trypsin treatment showed bands of molecular mass between $38-54 \mathrm{kDa}$ and $40-46 \mathrm{kDa}$, respectively. When tested with molecular subunits, pronase had less overall effect on structure
(Fig. 6c) than did trypsin (Fig. 6d). Chymotrypsin abolished virtually all antigenic reactivity. Some of the dominant bands of the ASS fraction (80-93 kDa), were consistently resistant to hydrolysis, being unaffected by 
all the enzymes tested. This was in marked contrast to the results seen with intact antigenic molecules of this fraction.

\section{Inhibition of antigen/antibody binding by lectins}

A. fumigatus transblotted antigens (WS-NCPF 2109 and fractions) were allowed to react with either ConA or WGA prior to incubation with anti-Aspergillus antisera. No reduction in antigen/antibody binding was apparent, relative to controls incubated in the absence of lectin.

An attempt was made to determine the extent to which protein and carbohydrate integrity of the glycoprotein molecules was a requirement for antigen/antibody binding. Transblotted antigens subjected to proteolytic degradation were subsequently incubated with lectin (ConA or WGA) conjugated to peroxidase. In neither case was measurable binding detected.

\section{Discussion}

Partially purified mycelial fractions of $A$. fumigatus, used in many laboratories as diagnostic antigens, still require full characterization (Schønheyder \& Andersen, 1984; Chaparas et al., 1986; Hearn et al., 1989; Kurup et al., 1989). The complex protein and glycoprotein mixtures separated here on gels and blots proved antigenically reactive over a wide range of molecular mass.

Slight cross-reactivity between $A$. fumigatus and other fungal classes, especially Coccidioides immitis, was apparent when tested in the immunoblot system with an $A$. fumigatus antiserum from rabbit. Their wall structures may contain at least two low molecular mass components which are common. In this regard the earlier report of two common antigens present in the walls of Coccidioides immitis and $A$. fumigatus is of interest (Cole et al., 1985). More definitive proof of the presence of shared antigenic components, by probing with antiserum pre-absorbed with heterologous antigens, was not sought.

Bands with apparent molecular mass between 93$95 \mathrm{kDa}$, occurring predominantly in the WS and AS75 material, reacted with an antiserum to a chymotryptic fraction. Harvey \& Longbottom (1987) have described a chymotryptic antigen (antigen 13) as ConA-binding and sized on Sephacryl S-200 as a $70 \mathrm{kDa}$ moiety. Any relationship between the antigens seen in the WS and AS75 fractions and this ' $\mathrm{C}$ '-antigen is speculative at this stage. It is known that the carbohydrate content of a glycoprotein can cause altered electrophoretic mobilities in SDS-PAGE giving anomalous results (Andrews, 1965; Bretscher, 1971). Therefore, any attribution of molecular mass values to these glycoprotein subunits is tentative, as their degree of glycosylation is unknown.

Reports from other laboratories have suggested an immunodominant role for galactofuranose residues in Aspergillus antigenic fractions (Sakaguchi et al., 1968; Reiss \& Lehmann, 1979; Bennett et al., 1985). In experiments described here, oxidation of sugar residues by sodium periodate treatment destroyed the antibodybinding capacities of most components present in the $A$. fumigatus fractions. Destruction was achieved using relatively mild conditions and suggests the presence of furanose, rather than pyranose sugars in these structures. Pre-incubation with either ConA or WGA of blotted antigens, which bind extensively to these lectins, failed to inhibit antigen/antibody binding. This suggests that neither mannose, glucose or hexosamine residues are directly involved in the antigen-binding site and provides indirect evidence in support of an immunodominant role for galactofuranosyl sugars.

However, with relatively ill-defined components and the use of polyclonal antibodies as probes, it is impossible to say with certainty which sugars are directly involved in the antigen-binding sites.

Differences between intact molecules and their subunits, in their susceptibility to enzyme attack, were most pronounced when exposed to the action of broadspectrum proteases. Pronase and papain exerted maximum effect on the intact molecules of WS and its fractions; blotted subunits were less prone to hydrolysis by these enzymes. Chymotrypsin and trypsin were destructive towards both types of structural moieties, although somewhat less capable than pronase and papain of the hydrolysis of intact molecules.

The effects of proteolysis on total structure were such that ConA and WGA are subsequently unable to bind to any glycoprotein molecules in these fractions indicating a considerable lack of molecular organization.

The polysaccharidases, principally Cytophaga lytic enzyme with specificity for $\beta$-1,3- and $\beta$-1,4-linked glucans, also disrupted antigen/antibody binding, mainly of the molecular subunits of $\boldsymbol{A}$. fumigatus. However, Cytophaga lytic enzyme is a crude mixture containing many activities; therefore hydrolysis by this extract should be interpreted with caution.

Anaylsis of WS mycelial antigens of several strains of A. fumigatus confirmed the immunological reactivity of glycoprotein molecules which occur in the wall and cytosol. The majority of these components of WS were sensitive to periodate oxidation and to hydrolysis by selected proteases. The importance of both carbohydrate and protein residues to antigen/antibody binding was demonstrated.

\section{References}

ANDREws, P. (1965). The gel-filtration behaviour of proteins related to their molecular weights over a wide range. Biochemical Journal 96, 595-606. 
Bennett, J. E., Bhattachar Jee, A. K. \& Glaudemans, C. P. J. (1985). Galactofuranosyl groups are immunodominant in Aspergillus fumigatus galactomannan. Molecular Immunology 22, 251-254.

BRETSCHER, M. S. (1971). Major human erythrocyte glycoprotein spans the cell membrane. Nature New Biology 231, 229-232.

Calvanico, N. J., Dupont, B. L., Huang, C. J., Patterson, R., Fink, J. N. \& KURUP, V. P. (1981). Antigens of Aspergillus fumigatus. I. Purification of a cytoplasmic antigen reactive with sera of patients with Aspergillus-related disease. Clinical and Experimental Immunology 45, 662-671.

Chaparas, S. D., Morgan, P. A., Holobaugh, P. \& Kim, S. J. (1986). Inhibition of cellular immunity by products of Aspergillus fumigatus. Journal of Medical and Veterinary Mycology 24, 67-76.

Cole, G. T., Chinn, J. W., Pope, L. M. \& Stark, P. (1985). Characterization and distribution of 3-O-methylmannose in $\mathrm{Cocci}^{-}$ dioides immitis. In Proceedings of the 4th International Conference on Coccidioidomycosis, pp. 130-145. Edited by H. E. Einstein \& A. Catanzaro. Washington, DC: National Foundation for Infectious Diseases.

Debeaupuis, J. P., Sarfat, J., Goris, A., Stynen, D., Diaquin, M. \& LATGÉ, J. P. (1990). Exocellular polysaccharides from Aspergillus fumigatus and related taxa. In Modern Concepts in Penicillium and Aspergillus Classification. Edited by R. A. Samson \& J. I. Pitt. New York \& London: Plenum Press (in the Press).

DEWAIR, M. \& BAUR, X. (1984). Radioallergosorbent test (RAST) for measurement of $\mathrm{IgG}$ antibodies to Aspergillus fumigatus in sera of patients with different lung diseases. Journal of Immunological Methods 75, 117-128.

Dubois, M., Gilles, K. A., Hamilton, J. K., Rebers, P. A. \& Smith, F. (1956). Colorimetric method for determination of sugars and related substances. Analytical Chemistry 28, 350-356.

Dzandu, J. K., Deh, M. E., Barratt, D. L. \& Wise, G. E. (1984). Detection of erythrocyte membrane proteins, sialoglycoproteins and lipids in the same polyacrylamide gel using a double-staining technique. Proceedings of the National Academy of Sciences of the United States of America 81, 1733-1737.

HANCOCK, K. \& TsANG, V. C. W. (1983). India ink staining of proteins on nitrocellulose paper. Analytical Biochemistry 133, 157-162.

HARVEY, C. \& LONGBOTTOM, J. L. (1986). Characterization of a major antigenic component of Aspergillus fumigatus. Clinical and Experimental Immunology 65, 206-214.

HARVEY, C. \& LONGBOTTOM, J. L. (1987). Characterization of a second major antigen, $\mathrm{Ag} 13$ (antigen $\mathrm{C}$ ) of Aspergillus fumigatus and investigation of its immunological reactivity. Clinical and Experimental Immunology 70, 247-254.

HEARN, V. M. \& MACKENZIE, D. W. R. (1980a). Mycelial antigen from two strains of Aspergillus fumigatus: an analysis by two-dimensional immuno-electrophoresis. Mykosen 23, 509-562.

HEARN, V. M. \& MACKENZIE, D. W. R. (1980b). Antigenic activity of sub-cellular fractions of Aspergillus fumigatus. Zeitblatt für Bakteriologie, suppl. 8, Medical Mycology, 173-181.

Hearn, v. M., Wilson, E. V., Proctor, A. G. \& Mackenzie, D. W. R. (1980). Preparation of Aspergillus fumigatus antigens and their analysis by two-dimensional immuno-electrophoresis. Journal of Medical Microbiology 13, 451-458.

HeARN, V. M., GRIFfiths, B. L. \& GoRIN, P. A. J. (1989). Structural analysis of water-soluble fractions of Aspergillus fumigatus mycelium. Glycoconjugate Journal 6, 85-100.
JOHN, J., WILSON, E. V. \& HEARN, V. M. (1984). Analysis of Aspergillus fumigatus germ tube surface structures by an immuno-fluorescent labelling technique. Mykosen 27, 485-497.

Kurup, V. P., Greenberger, P. A. \& Fink, J. N. (1989). Antibody response to low-molecular-weight antigens of Aspergillus fumigatus in allergic bronchopulmonary aspergillosis. Journal of Clinical Microbiology 27, 1312-1316.

LAEMMLI, U. K. (1970). Cleavage of structural proteins during the assembly of the head of bacteriophage T4. Nature, London 227, 680685.

De Magaldi, S. W. \& Mackenzie, D. W. R. (1984). Specificity of antigens from pathogenic Aspergillus species. I. Studies with ELISA and immuno-fluorescence. Sabouraudia 22, 381-394.

MoNK, B. C. (1987). Electrotransfer of SDS-PAGE separated polypeptides to the DE81 blotting matrix and detection of Chlamydomonas antigens and glycoconjugates. Journal of Immunological Methods 96, 19-28.

PROCTOR, A. G. (1976). Mycological methods. In Microbiological Methods, 4th edn, pp. 181-193. Edited by C. H. Collins \& P. M. Lyne. London: Butterworths.

Ramirez, P., Bonilla, J. A., Moreno, E. \& Leon, P. (1983). Electrophoretic transfer of viral proteins to nitro cellulose sheets and detection with peroxidase-bound lectins and protein A. Journal of Immunological Methods 62, 15-22.

READ, S. M. \& NORTHCOTE, D. H. (1981). Minimization of variation in the response to different proteins of the Coomassie Blue $G$ dyebinding assay for protein. Analytical Biochemistry 116, 53-64.

Reiss, E. \& LehmanN, P. F. (1979). Galactomannan antigenemia in invasive aspergillosis. Infection and Immunity 25, 357-365.

SAKAGUCHI, O., SUZUKI, M. \& YoKoTA, K. (1968). Effect of partial acid hydrolysis on precipitin activity of Aspergillus fumigatus galactomannan. Japanese Journal of Microbiology 12, 123-124.

SCHøNHEYDER, H. \& ANDERSEN, P. (1984). IgG antibodies to purified Aspergillus fumigatus antigens determined by enzyme-linked immunosorbent assay. International Archives of Allergy and Applied Immunology 74, 262-269.

Steinbuch, M. \& Audran, R. (1969). The isolation of IgG from mammalian sera with the aid of caprylic acid. Archives of Biochemistry and Biophysics 134, 279-284.

Towbin, H., StaeHELIN, T. \& Gordon, J. (1979). Electrophoretic transfer of proteins from polyacrylamide gels to nitrocellulose sheets: procedure and some applications. Proceedings of the National Academy of Sciences of the United States of America 76, 43504354.

Tran van Ky, P., Biguet, J. \& Fruit, J. (1966). Localisation et fréquence des arcs des immuno-électrophoregrammes produits de la serum des malades atteints de mycetomes aspergillaires appliquées contre l'antigene Aspergillus fumigatus. Revue d'Immunologie, 30, 1320.

Wilson, E. V. \& HeARn, V. M. (1983). Use of Aspergillus fumigatus mycelial antigens in enzyme-linked immunosorbent assay and counter-immunoelectrophoresis. Journal of Medical Microbiology 16, 97-105.

Wilson, E. V., Hearn, V. M. \& Mackenzie, D. W. R. (1987). Evaluation of a test to detect circulating Aspergillus fumigatus antigen in a survey of immunocompromised patients with proven or suspected invasive disease. Journal of Medical and Veterinary Mycology 25, 365-374. 